\title{
Effect of Scoliosis on Growth of Alveoli and Pulmonary Arteries and on Right Ventricle
}

\author{
G. DAVIES* and LYNNE REID \\ From the Department of Experimental Pathology, Institute of Diseases of the Chest, Brompton Hospital, London
}

\begin{abstract}
Davies, G., and Reid, L. (1971). Archives of Disease in Childhood, 46, 623. Effect of scoliosis on growth of alveoli and pulmonary arteries and on right ventricle. During childhood the lung not only grows in size but alveoli and arteries multiply and the pattern of muscularity of the arteries changes. A quantitative study is here reported on 4 cases of scoliosis. The limitation and distortion of space available affect lung growth. In scoliosis, alveoli are too few in number, often emphysematous, and may even atrophy, the changes being irregularly distributed throughout a lobe or lung. Generally, the size of the large pulmonary arteries is appropriate to the lobar volume and hence small for the age of the child: arterial muscle hypertrophy and abnormal extension to the periphery were seen only in 2 of the 4 cases in which there was right ventricular hypertrophy. In these, hypoxaemia had been present for some time before death and this, not hypoplasia, seemed responsible for the muscle hypertrophy. In 2 cases where scoliosis was associated with a mesodermal dysplasia, there was an excessive number of intra-acinar arteries.
\end{abstract}

It has been known for centuries that deformity of the spine may cause difficulty with respirationindeed, Hippocrates recognized it. As early as 1846, Latham, in his lectures on the heart, commented that curvature of the spine may, by deforming the chest, give rise to cardiac failure. Interest in cardiac failure in scoliosis revived in this country in 1930, when Coombs published an account of four fatal cases. In 1939, Chapman, Dill, and Graybiel gave a full clinical account of scoliosis complicated by heart failure.

Pathological study of the small pulmonary arteries in scoliosis was initiated by Lewis et al. (1952). They studied one case in detail and found thickening of the walls of the pulmonary arterioles, but Fischer and Dolehide (1954) disagreed, finding that the small pulmonary arteries were normal in appearance. Neither group of authors had injected the arterial system. Bergofsky, Turino, and Fishman (1959), in the course of a detailed physiological study of scoliosis, examined the pulmonary arteries in a few cases in which the arterial tree had been injected (though no details of technique are mentioned) and showed hypertrophy and hyperplasia of the

\footnotetext{
Received 5 April 1971.

^Present address: Sunnybrook Hospital, 2075 Bayview Avenue, Toronto 12, Canada.
}

arterial media. They also found the alveoli to be small, with only occasional areas of mild alveolar dilatation, but again the lungs were not inflated.

Naeye (1961) measured medial muscle mass, using a planimeter to assess medial area, in 9 cases of scoliosis showing clinical signs of pulmonary hypertension. He confirmed the increase in the arterial muscle, and considered that the change was due to hypoxaemia.

The methods Elliott (1964) had used in his study of the normal adult lung were applied by Reid (1966) to the lungs of scoliotic patients. The number of small arteries counted in a given area of lung section was related to the number and size of the alveoli, as estimated by a linear intercept method. It became clear from injection studies that in patients whose scoliosis had become prominent before the alveoli were numerically fully developed-that is, before the age of 8 years (Dunnill, 1962)-there was hypoplasia of lung, both of alveoli and of arteries. Though hypoplasia was a major finding, atrophy was also seen in lung overlying the apex of a severe curve. Collapse, on the other hand, was not found, nor was congestion.

In scoliosis several mechanisms have been put forward at one time or another to explain the fre- 
quent finding of hypertrophy of the right ventricle that often progresses to heart failure: collapse, congestion, the small size of the pulmonary vascular bed, arterial medial hypertrophy, hypoxaemia, atrophy, and hypoplasia.

The interest in the effects of scoliosis on the development of the lung lies in the limitation of space available for lung growth. The disease develops after birth, and may affect the lung during its period of most rapid growth, the first $\mathbf{8}$ years of life. It is not likely to affect the development of the bronchial tree, as this is fully formed by the 16th week of intrauterine life (Bucher and Reid, 1961), but it may well interfere with the development both of the alveoli, which form after birth, and of the small pulmonary arteries, which multiply and are remodelled after birth (Davies and Reid, 1970). There may also be an effect on the right ventricle, the hypertrophy of which may be detected by ECG or by weighing the ventricles post mortem.

The objects of this study were to investigate the effect of scoliosis on (i) the growth of the alveoli, (ii) the size and number of the pulmonary arteries, and (iii) the distribution of muscle tissue within the pulmonary arteries. The changes in the alveoli and arteries might then be related to the development of right ventricular hypertrophy.

\section{Material and Methods}

Six lungs and two hearts were examined from four cases of scoliosis, aged $8,15,15 \frac{1}{2}$, and 18 years. These cases are listed in Table $I$ and will be referred to as Case, S1, S2, S3, and S4, in order of age. The clinical histories are given in the Appendix. Cases S1, S2, and S4 died from cardiorespiratory failure, and S3 died an accidental death.

TABLE

Material Examined from Four Cases of Scoliosis

\begin{tabular}{|c|c|c|c|c|c|}
\hline \multirow{2}{*}{ Case } & \multirow{2}{*}{$\begin{array}{l}\text { Age } \\
\text { (yr) }\end{array}$} & \multirow{2}{*}{ Sex } & \multicolumn{3}{|c|}{ Organs Available } \\
\hline & & & $\begin{array}{l}\text { Right } \\
\text { Lung }\end{array}$ & $\begin{array}{c}\text { Left } \\
\text { Lung }\end{array}$ & Heart \\
\hline $\begin{array}{l}\text { S1 } \\
\text { S2 } \\
\text { S3 } \\
\text { S4 }\end{array}$ & $\begin{array}{l}8 \\
15 \\
15 \frac{1}{2} \\
18\end{array}$ & $\begin{array}{l}\mathbf{M} \\
\mathbf{F} \\
\mathbf{F} \\
\mathbf{M}\end{array}$ & $\begin{array}{l}+ \\
+ \\
+ \\
-\end{array}$ & $\begin{array}{l}+ \\
+ \\
+\end{array}$ & $\begin{array}{l}+ \\
+ \\
- \\
-\end{array}$ \\
\hline
\end{tabular}

The methods employed in the study of these lungs and hearts have been described in a previous paper (Davies and Reid, 1970), in which a series of normal children were studied as an essential preliminary to the work reported here.

\section{Results}

Lung and lobar volumes.* All the lungs were smaller than normal for age, and the degree of reduction in volume of individual lobes variedfor instance, in Case S1 the middle lobe was the largest of the three lobes of the right lung. The ratio between lobar volumes in the right lung, if as predicted from the number of segments in each lobe, is RUL : RML : RLL $=0 \cdot 3: 0 \cdot 2: 0 \cdot 5$; and in the left lung LUL : LLL $=0.5: 0.5$.

The most extreme deviations from these predicted values were seen in Case $S 1$ where the ratios for the three lobes of the right lung were $0 \cdot 4,0 \cdot 4$, and 0.2 respectively, and for the two lobes of the left lung 0.7 and 0.3 respectively. Because of the small lung volumes in these cases, when describing the effects of scoliosis on the alveoli and pulmonary arteries, comparison has been drawn not only between normal and diseased lungs of similar age, but also between normal and diseased lungs of similar volume.

Lung shape. None of the lungs was of normal shape, and some were grossly distorted. Even after inflation and fixation the lung maintained the shape of the thorax. In some cases, for instance the left lung of Case S4, the lung was rotated about its vertical axis by as much as $45^{\circ}$. The medial surface of some upper lobes showed a deep indentation from a displaced aorta, while in other specimens an impression of the vertebral column was often visible on the posteromedial surface. In Fig. 1 the fixed and inflated lungs of Case S1 are illustrated.

Macroscopical examination. The uneven effect of the disease even within a single lobe could often be seen by comparing the size of the acini, identifiable from the distribution of the small arteries either as seen through the pleura or on the cut surface; an area containing small acini might be seen close to an area containing larger acini (see Fig. 2). In many areas the arteries close to the pleural surface were disproportionately large, suggesting loss of the superficial layer of lung tissue.

\section{Microscopical examination}

Alveolar measurements. Throughout all lobes of all lungs there was some variation in alveolar size; this was not extreme, but the number included in successive fields in some lobes varied by as much

\footnotetext{
${ }^{\star}$ Results reported in this paper are available in tabular form from L.R.
} 


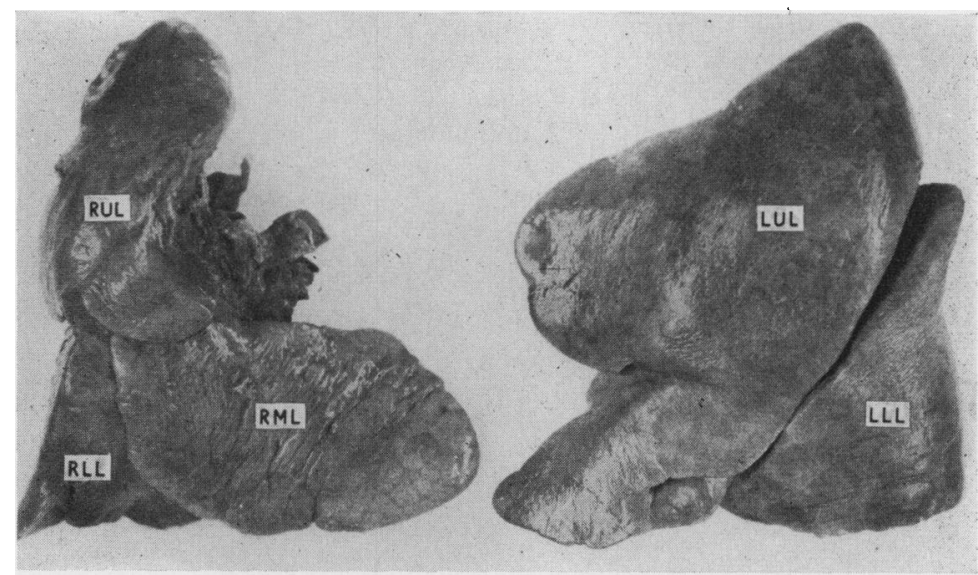

FIG. 1.-Case S1. External view of lungs to show distortion produced by scoliosis: the left upper lobe (LUL) is larger than the left lower $(L L L)$, the right upper lobe $(R U L)$ and lower lobes $(R L L)$ are extremely small, the right middle $(R M L)$ larger than the right lower.

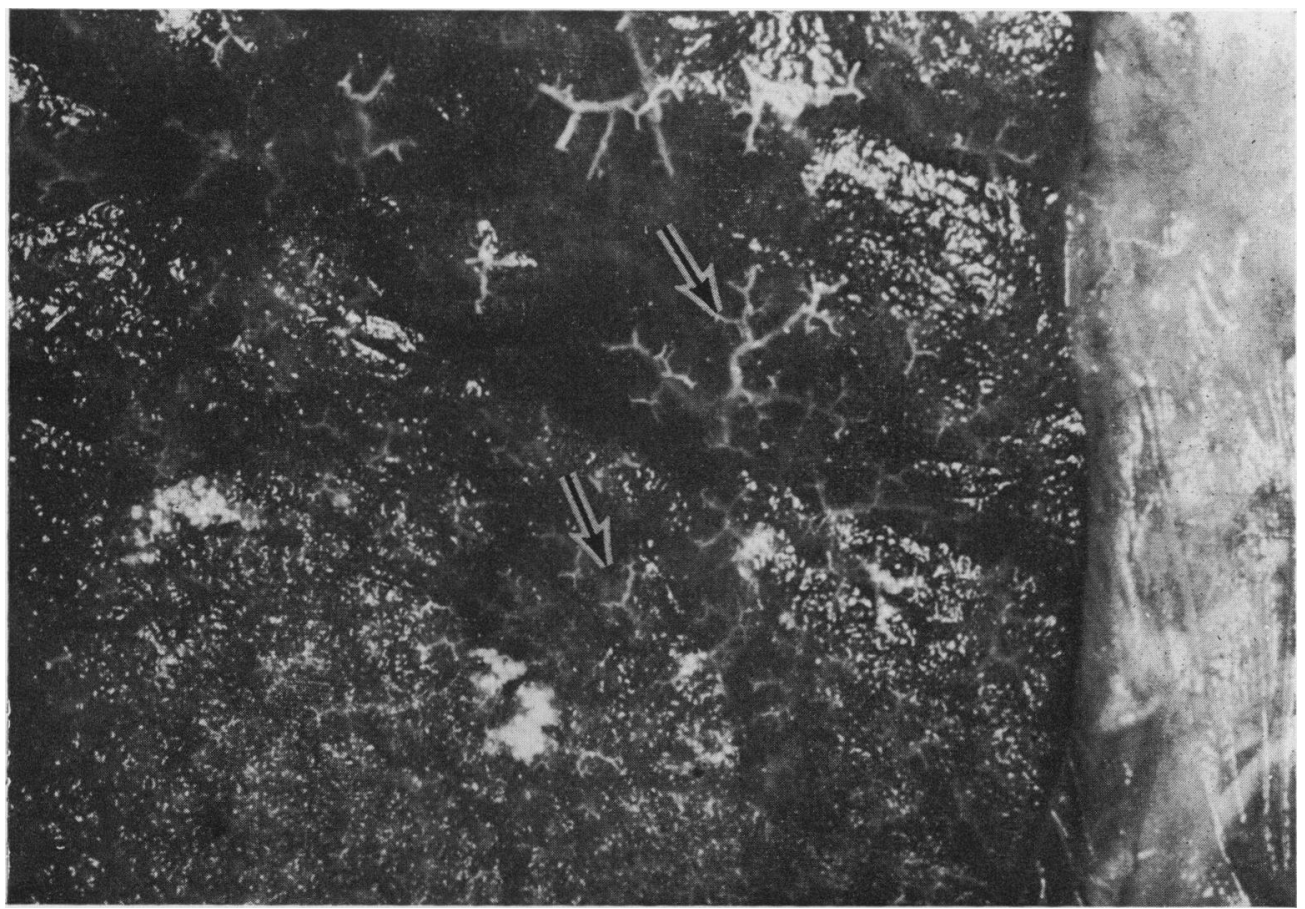

Fig. 2.-Case S4. Lateral view of the lower lobe after the pleura (to the right) had been peeled off to show the pattern of subpleural acinar arteries. The upper arrow points to a large acinus, the lower to one that has failed to develop normally.

as $40 \%$. In order to determine mean alveolar dimensions, a larger number of fields had to be examined in each lobe than was necessary in normal lungs.

Alveolar number. In all lobes the alveoli were fewer in number than would be expected from the patients' ages. In Case S1, though the patient was 8 years old, the total alveolar number was that of a child of about 4 months of age. In Case S2, a girl of 15 years, the number was appropriate to a child of 1 year; in Case S3, a girl of $15 \frac{1}{2}$, to a child 


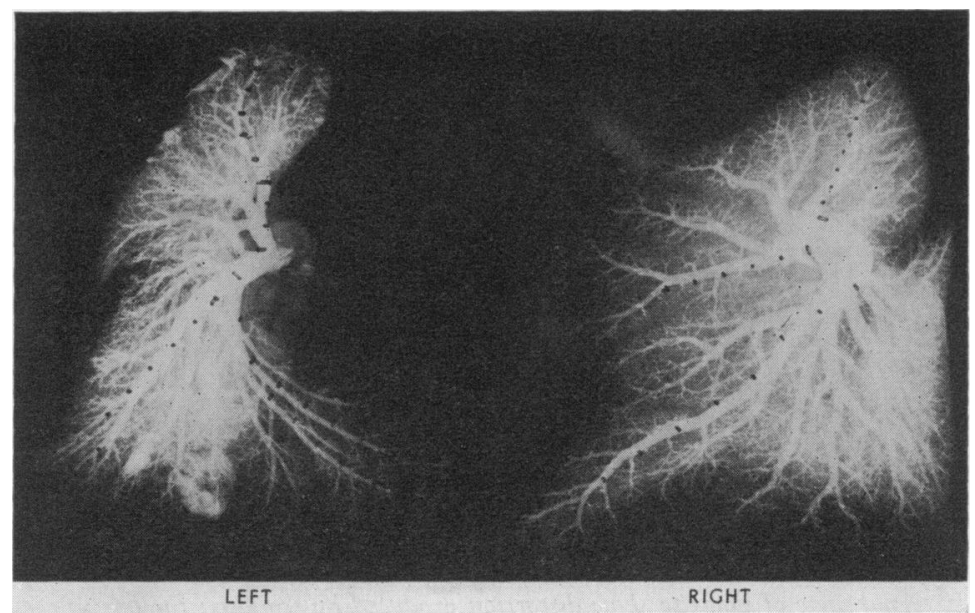

FIG. 3.-Case S1. Arteriograms of both lungs to correspond with Fig. 1 showing the irregular development through the lungs with crowding and hypoplasia of vessels in many areas.

of 18 months; and in Case S4, a boy of 18 , to a child of about 3 months.

Within any one lung the lobes were unevenly affected, some having relatively fewer alveoli than others. Generally speaking, the lobes most reduced in volume contained fewest alveoli. The alveoli in most lobes were also fewer in number than was expected from the lobar volumes, which suggests that the mean alveolar size was too large.

Alveolar size. The mean size of the alveoli in each lobe also varied. In 14 of the 15 lobes the alveoli were abnormally large for the lobar volume; in the one exception (the right lower lobe of case S1) the mean alveolar size was appropriate to lobar volume, but this lobe was the most reduced in volume of any lobe studied. In most lobes the alveolar size was roughly appropriate to the age of the patient, so that, despite the reduction in number the alveoli had continued to grow in volume. In a few lobes they were a little larger than would be normal for age.

Alveolar shape. The degree of complexity of shape of the alveoli was usually simpler than would be expected, though occasionally normal; never was the outline more complex than was expected.

Right ventricular weight. In only 2 cases was the heart available for examination.

In Case S1 the total heart weight was $80.5 \mathrm{~g}$; the left ventricle and septum $(\mathrm{LV}+\mathrm{S})$ together weighed $54 \mathrm{~g}$ and the right ventricle (RV) $26.5 \mathrm{~g}$, giving a ratio $(L V+S) / R V$ of $2 \cdot 02$. This indicates a mild degree of right ventricular hypertrophy (RVH); the range in normal adults is 2.3-3.3: 1 (Fulton, Hutchinson, and Jones, 1952).

In Case S2 the total heart weight was $218.5 \mathrm{~g}$, $\mathrm{LV}+\mathrm{S} 129 \mathrm{~g}, \mathrm{RV} 89 \cdot 5 \mathrm{~g}$, giving a ratio $(\mathrm{LV}+\mathrm{S})$ / RV of 1.44 . This indicates a severe degree of right ventricular hypertrophy.

In Case S3 the coroner's pathologist reported a normal heart, with no evidence of right ventricular hypertrophy on inspection of the thickness of the wall of the right ventricle. In Case S4, the necropsy report referred to 'mild hypertrophy' of the right ventricle as determined by inspection of its wall.

\section{Measurement of pulmonary arteries}

Arteriograms. In all arteriograms the size of the pulmonary arteries was small but appropriate to the volumes of the lungs. There was some tortuosity of axial arteries in lobes of grossly distorted shape, and in many areas the arteries near the pleura were too large and branched at abnormal angles. Such changes may be seen in the arteriogram of Case S1, illustrated in Fig. 3.

The peripheral arterial filling was dense in 2 cases, S1 and S3, though the density of filling varied from area to area within a lobe. In Cases S2 and S4 the peripheral filling was less dense but not sparse; in each case there were a few areas in which filling was dense. In the left lung of Case S3 the pulmonary venous system was injected under high pressure before the arterial system was injected, so that a combined venogram and arteriogram was prepared. This led to very dense filling 
Cose SI. Right and left orteriogrom.

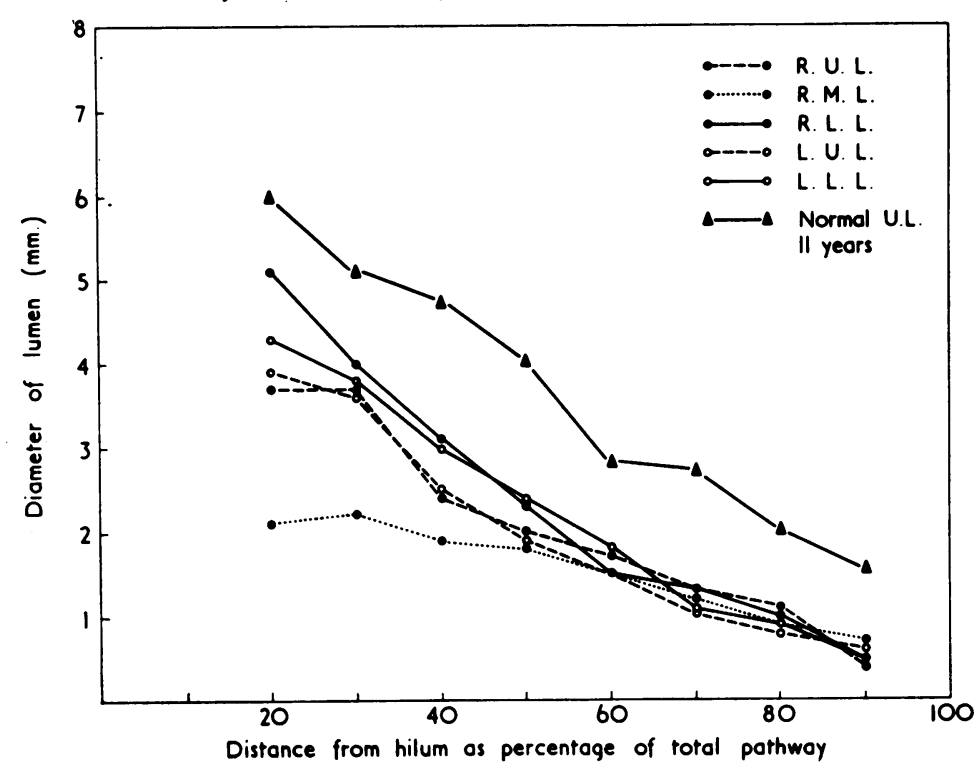

Fig. 4.-Case S1. Measurements on the arteriogram of each of the five lobes. All are smaller than a pathway in the left upper lobe of the same age. ( $A$ lower lobe would be so large that it would not be included on this diagram.)

of fine vessels, but it proved impossible to differentiate with confidence between arteries and veins either in the angiogram or on microscopical examination of the lung.

The diameter of the pulmonary arteries as measured from the arteriograms was usually appropriate to the volumes of the lobes, so that the largest lobes contained the largest proximal and mid lung arteries. This did not hold in a few lobes, notably the very small right lower lobe of Case S1, in which the pulmonary arteries were inappropriately large for the lobar volume (see Fig. 4). It might be that this appearance indicates atrophy of the lobe following more normal development. Despite the difference from lobe to lobe in the size of the more proximal arteries, near the pleural surface the arteries were of similar diameter, save when the artery happened to run to an area of atrophic lung tissue.

Number of small arteries in unit area of lung. In no case was there any marked reduction of the number of arteries between 25 and $200 \mu \mathrm{m}$ in diameter within a unit area of lung. In Cases S1 and S3 (right lung only, as the venous system had also been filled in the left lung), the number of small arteries was greatly increased. Numerous small non-muscular arteries lay in alveolar walls, often in clumps, and sometimes completely sur- rounded an alveolar space. This appearance was seen in no other lung in this series, nor in other lungs of adults and children examined in this laboratory. The explanation can only be speculative; in both cases the cause of the scoliosis was a mesodermal abnormality-in Case S1 a muscular dystrophy, in Case S3 a hemivertebra-and it may be that some factor peculiar to those conditions was operating.

In Case S2, though the arteries between 50 and $200 \mu \mathrm{m}$ in diameter were a little reduced in number below the normal, the smaller arteries from 25 to $50 \mu \mathrm{m}$ in diameter were increased. In Case S4 the number of arteries was probably appropriate to the boy's age and alveolar size.

Size range of different types of artery, and population counts. The diameter range of the three types of pulmonary artery described-non-muscular, partially muscular, and wholly muscular was analysed. In Cases $S 1$ and S2 muscle extended into smaller arteries than would be normal for age. This is illustrated by the population distribution curve in Case S1 (Fig. 5); the curve lies further to the left than would be normal. The muscle extended in both cases into arteries smaller than would be appropriate to the lung volumes, or to the age of the patient. In Case S3 muscle tissue was found only in arteries which were larger than 


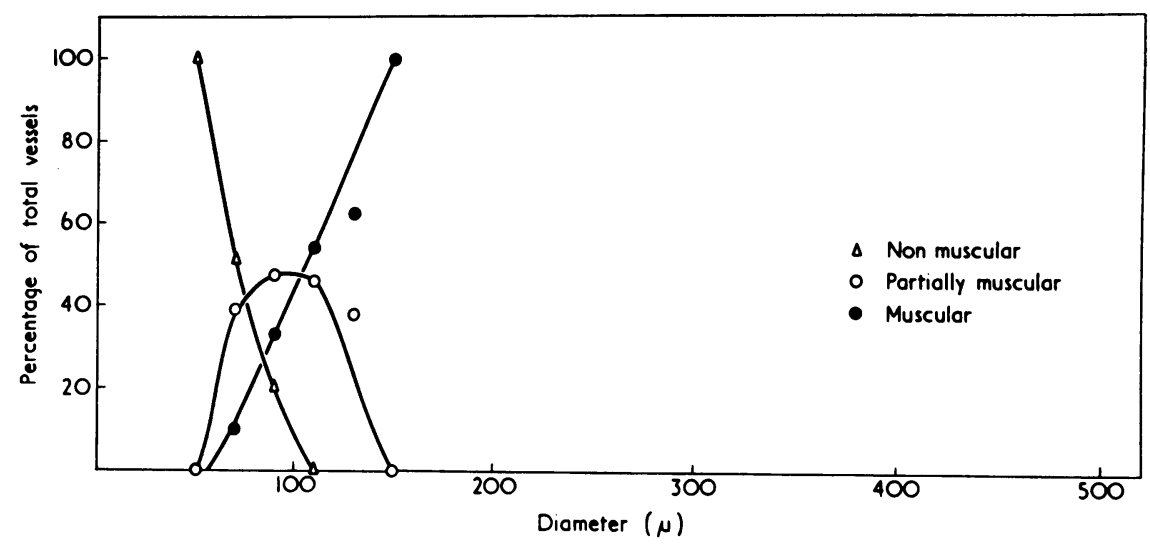

FIG. 5.-Case 1. A 'population count' in this 8-year-old patient shows a distribution of the various structural types of artery which is similar to that in a newborn or in an adult.

any of those in the normal children examinedarteries, with incomplete muscular coats, as large as $450 \mu \mathrm{m}$ in diameter were found (Fig. 6). This is certainly inappropriate to the child's age, but might be appropriate to a child aged between 5 and 11 years. In Case S4 muscle extended into arteries almost as small as was seen in the adultthe distribution is probably appropriate to a child of rather less than 18 years.

In Cases S1 and S2, therefore, muscle tissue had passed further into the acinus than normal, while in Cases S3 and S4 the normal peripheral growth of muscle appeared to have been retarded.

Percentage wall thickness. Again, Cases S1 and S2 showed the greatest degree of arterial wall thickening. The percentage wall thickness ( $\%$
WT) was raised in all size ranges of arteries (see Fig. 7) and the variation about the mean was wide.

The arteries in Case S3 had walls of normal thickness, the mean values lying well within the normal adult range, and the variation from the mean was small. Measurement of the \%WT of arteries and veins in the left lung produced figures similar to those found in the right lung, with no greater variation from the mean. In Case S4 the mean \%WT was a little high in all diameter ranges.

Index of medial area. The index of medial area was estimated for each of the 13 lobes studied. High figures in Cases $S 1$ and S2 confirm the hypertrophy of muscle tissue in the small pulmonary arteries in these 2 cases. In Case S3 the figures are low, reflecting the recession of muscle tissue

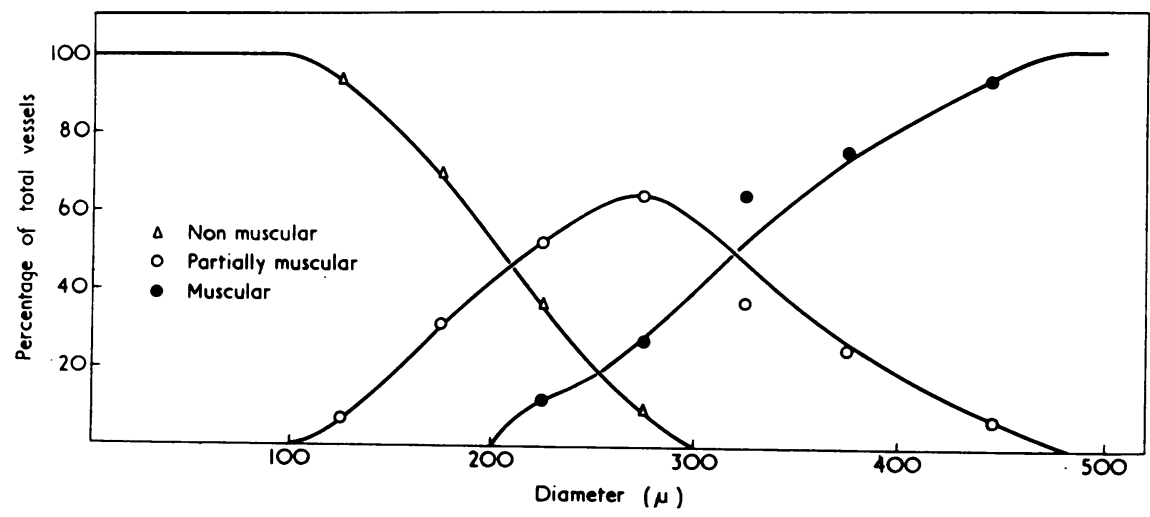

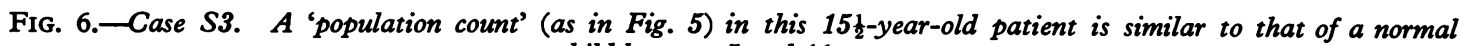
child between 5 and 11 years. 


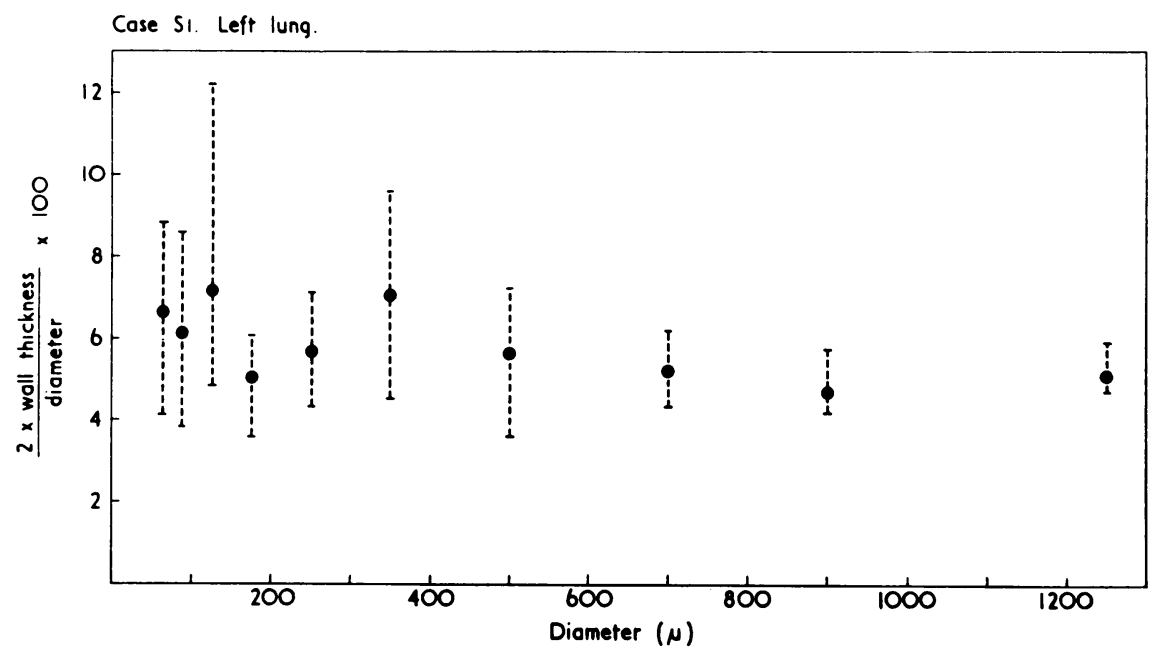

FIG. 7.-Case S1. Left lung-increased wall thickness of the pulmonary arteries is seen, together with abnormal variability of thickness. At this age the normal value for the index of wall thickness, in terms of the units shown on the vertical axis, is between 1 and 2.

into larger arteries. In Case S4 the figures may be normal for age.

Arteries running with terminal and respiratory bronchioli. The mean diameter and range of arteries running with terminal and respiratory bronchioli in these cases, and the percentage of arteries that were partially muscular in type was also calculated. In each case the arteries were small for age, but they were appropriate in size to the lung volumes in Cases S1, S3, and S4, while in Case S2 they were only a little smaller than would be expected from the lung volume.

In each case all the arteries running with terminal bronchioli were wholly muscular, which was to be expected for the patient's ages. In Case S1, the arteries running with respiratory bronchioli were all wholly muscular in type; in a normal child of this age some of these arteries will have partially muscular coats. This is further evidence of the peripheral extension of arterial muscle in this case. In Case S2 all arteries with respiratory bronchioli were wholly muscular, but this could be normal for the age of 15 years. Case S3, however, was much the same age, and here some of these arteries were partially muscular in type, reflecting the absence of muscle tissue in quite large arteries. In Case S4, as would be expected at this age, all these arteries were wholly muscular.

\section{Discussion}

Effect of scoliosis on alveolar development. In all lobes from all 4 cases the alveoli were few in number. This could be due to underdevelopment, to atrophy, or to both. It is clear from Case S4 that an overall atrophy may play a part in the reduction of alveolar number. The total number of alveoli was considerably less than would be found in a child of 5 years, and it may be assumed that this boy's lungs were normal at least until his attack of poliomyelitis at the age of 5 years.

Macroscopical examination of the lungs shows that atrophy may lead to a local loss of alveoli, as in some areas where local conditions in the thorax had led to indentations in the surface of the lung, large arteries lay close to the pleura and the pattern of fine intra-acinar branching was lost, indicating loss of the superficial layer of acinar tissue. This effect, however, was seen only on the surface of the lung, whereas the alveoli were reduced in number throughout.

Increasing deformity may be expected to lead to progressive distortion of the lung, and this might compress the alveoli; atrophy of alveolar walls might then take place. Compression should lead to small alveoli with complex, folded walls, but since nearly all the alveoli found were large in size and of normal or simple outline, it is probable that compression of lung tissue is not a major factor in reducing the number of the alveoli.

Evidence of underdevelopment of alveoli is more difficult to find. If space for development were the limiting factor in alveolar multiplication, then the alveolar number should be at least appropriate to the lung volume, but in all cases examined the alveoli were fewer. If limitation of space acts as 
a brake tc alveolar multiplication, it cannot be the only factor, as multiplication was diminished more than was accountable by limitation of space.

It is interesting to compare the alveoli in these cases with those described by Areechon and Reid (1963) in the lungs of two children with congenital diaphragmatic hernia dying soon after birth. Though conclusions drawn from alveoli in the newborn may be misleading, the alveoli in the cases of diaphragmatic hernia were found to be reduced in size rather than in number per acinus. Limitation of space for lung development in utero had, therefore, little effect on multiplication of the alveoli unless, as Areechon and Reid suggested, alveolar development started rather earlier in intrauterine life than is normal. This has been confirmed in a later case using quantitative analysis (Kitagawa et al., 1971).

In Cases S1, S2, and S3 the number of alveoli had increased after birth, but appeared to have checked early in life, though they had grown to more or less normal size and shape. It is probable that both underdevelopment and atrophy were responsible in each case.

Effect of scoliosis on pulmonary arterial size and number. Generally speaking, the pulmonary arteries were appropriate in size to the lobar volumes-this was confirmed both by inspection and measurement of the arteriograms, and by the size of the arteries running with terminal and respiratory bronchioli. All the lobes were small for age, which implies that all the pulmonary arteries were small for age. In some cases, best exemplified by Case $S 1$, the arteries appeared not to have grown to a size compatible with age, but in Case S4, the arteries were smaller than in a normal 5 -year-old, and here the arteries must have decreased in size as the lung grew smoller with advancing scoliosis. This provides evidence both of underdevelopment and of atrophy of the pulmonary arteries. Locally areas of atrophy of the peripheral portion of the arterial tree could be seen macroscopically, especially where there were indentations in the lung surface.

The intra-acinar arteries were not reduced in number in any case. In Case S2 the reduction in number of arteries of 50-200 $\mu \mathrm{m}$ diameter appeared to be compensated by the increase in number of smaller arteries; in Case $S 4$ the arterial number was normal. In Cases S1 and S3 there were large numbers of intra-acinar arteries and it is believed that these vessels, occurring as they did in clumps, may represent an unexplained feature of mesodermal disease.
Effect of scoliosis on distribution of muscle tissue in pulmonary arteries. In 2 cases, $S 1$ and S2, there was evidence of hypertrophy of arterial muscle, in that it had extended further than normal towards the periphery of the lung, and that the muscular walls had become thickened. These arteries therefore presented the paradox of hypoplasia in size, together with hypertrophy of their muscle tissue.

It was in these 2 cases that signs and symptoms of hypoxaemia and cardiac failure had been present for a long period before death. It is possible that the hypoxaemia had promoted the growth of excess muscle tissue in the lungs; it is also possible that the muscle tissue extending further into the acinus had reduced the area of arterial wall available for free gas exchange, thereby encouraging further hypoxaemia.

In the other 2 cases, S3 and S4, there was evidence of hypoplasia of arterial muscle, as measured by the degree of extension of muscle to the lung periphery. The distribution of muscle was probably appropriate to a younger child. This was more marked in Case S3, in which the adolescent growth of new arterial muscle had failed to occur, so that muscle was absent in large arteries. In Case S4 the adolescent growth of muscle had taken place, but not quite to the extent to be expected at this age. The slight thickening of the arterial walls in this case may suggest that hyperplasia of muscle as a response to disease may partially have masked the full degree of hypoplasia of muscle as a response to failure of the arteries to grow in size.

It is suggested that scoliosis, by preventing growth in size of the arteries, causes the growth of new muscle, usually beginning between the ages of 5 and 11 years, to be delayed or retarded. In cases in which the blood-gas interface is so reduced as to lead to hypoxaemia, hypertrophy of arterial muscle may follow, leading to extension of muscle further into the acinus and arterial wall thickening.

Right ventricular hypertrophy in scoliosis. In two of the cases described, S1 and S2, there was right ventricular hypertrophy (RVH). In Case S4 there may have been mild RVH; in Case S3 there was none. In Cases S3 and S4 the respiratory disability from scoliosis had not been severe. This is usual at this age; it is exceptional for children to die of the respiratory complications of scoliosis, and with neither of these children was death due to chronic respiratory illness. There had been no prolonged period of cyanosis or cardiac failure in either case, and on examination of the lungs there was no hypertrophy of arterial muscle tissue. In 


\section{Effect of Scoliosis on Growth of Pulmonary Development}

Cases S1 and S2 respiratory disability had been so severe as to cause prolonged central cyanosis and heart failure, and arterial muscle hypertrophy was found in both cases.

The following hypothesis is advanced to account for the occurrence of RVH in scoliosis: the effect of scoliosis is (1) to prevent multiplication of the alveoli, and (2) to lead to alveolar atrophy. There follows a diminution of the total alveolar surface area and hence a decrease in the blood-gas interface. If this is severe, hypoxaemia in the pulmonary blood vessels may occur. Hypoxaemia leads to hypertrophy of arterial muscle; muscle tissue extends further into the acinus, thereby possibly worsening the hypoxaemia, and the arterial walls become thickened. This leads to a rise in pulmonary vascular resistance and hence in pulmonary arterial pressure, increasing the work load on the right ventricle and causing it to hypertrophy.

\section{APPENDIX}

\section{Clinical Histories of 4 Cases of Scoliosis}

Case S1. Male, aged 8 years at death. This boy was thought to be normal at birth. At 4 months he was noticed to have a right-sided weakness, which progressed until at 18 months he had acquired an equinus deformity of the right foot which was treated with a plaster splint. At $2 \frac{1}{2}$ years bilateral subluxation of the hips led to further treatment with splints, and at 3 years 8 months it was realized that he was suffering from a disease characterized by general muscular hypotonia. He was unable to walk, all his muscles were weak and flaccid, the central musculature being more severely affected than the peripheral musculature, and the facial muscles were almost useless. There was moderate scoliosis.

A muscle biopsy showed changes 'characteristic of a muscular dystrophy'.

The boy was allowed to return to his home, where he lived for the next 3 years, during which he was subject to frequent attacks of bronchitis, and was treated with courses of antibiotics. At the age of 7 years 10 months he was unable to sit or even to correct the position of his head without support; his face was expressionless and he was unable to close his mouth. There was extreme wasting and weakness of all muscles with severe flexion deformity of the elbows, hips, and knees, and the scoliosis was now severe. He was cyanosed at rest, but there were no signs of heart failure. His vital capacity was $200 \mathrm{ml}$. Despite the severe disease the boy was intelligent and co-operative. Chest $x$-ray showed a severe scoliosis convex to the right, the right lung being visible only as a thin band of air-containing tissue.

In the next few months he suffered from repeated respiratory infections, culminating in heart failure. His condition gradually deteriorated and he died, deeply cyanosed and in severe heart failure, at the age of 8 years 2 months.

The scoliosis therefore developed in the first 2 years of life and progressed steadily in severity. In all probability there was underventilation for many years, but cyanosis was first noticed about a year, and heart failure about 3 months, before his death.

Case S2. Female, aged 15 years at death. The early part of this girl's history is not known. She lived in Dominica until the age of 9, when she came to England. She is believed to have been deaf from birth or from very early childhood and was mentally retarded. She had, in early childhood, developed a kyphoscoliosis which was already very severe when she first came to this country, and its cause could not be determined.

At the age of 10 she developed a mild spastic paraplegia. This was relieved by laminectomy and dural decompression, which achieved complete recovery of spinal cord function.

At the age of 12 she was admitted to hospital with a respiratory infection, there being signs of pneumonic consolidation in the right lower chest. She was not centrally cyanosed, but there were signs of heart failure. There was a gross scoliosis convex to the right. There was an iron deficiency anaemia attributed to a whipworm (Trichuris) infection; this responded to treatment with mepacrine. After correction of the anaemia the girl was not cyanosed.

An ECG at this time showed evidence of right ventricular hypertrophy. Treatment with antibiotics and physiotherapy led to improvement in the chest condition, and the heart failure was controlled with digitalis and diuretics. Nine months later, when the girl was 13, she was again in heart failure, and was found to be cyanosed. The arterial $\mathrm{PCO}_{2}$ was $70 \mathrm{mmHg}$. Treatment in hospital led to some improvement in her condition, but the cyanosis remained, at first only on exercise, but, after a few months, at rest as well.

Supportive therapy was continued at a special school, and the girl remained out of hospital until shortly before her 15th birthday, when deepening of her cyanosis and worsening of her heart failure led to her readmission. During this period of treatment a right heart catheter was passed, and the pulmonary artery pressure was found to be $40 / 10 \mathrm{mmHg}$, mean value $19 \mathrm{mmHg}$. The indirect left atrial (pulmonary wedge) pressure was normal, and no sign was found of an intracardiac shunt. Systemic arterial oxygen saturation was $80 \%$.

A few months later the child died of acute respiratory infection.

This girl had suffered from a scoliosis of unknown cause from early childhood. She first developed chest infection and signs of heart failure 3 years before her death, and cyanosis was first seen 2 years before her death.

Case S3, Female, aged $15 \frac{1}{2}$ years at death. A detailed history of this girl's disease was not available, the lungs having been obtained from the coroner.

She had had scoliosis from early childhood, due to a hemivertebra present from birth. While this had caused severe deformity, the girl was not handicapped by dyspnoea, and at no time had she sought treatment 
for respiratory disease. She had never been cyanosed and had never been in heart failure. She died of acute coal gas poisoning.

Case S4. Male aged 18 years at death. This boy was well until the age of 5 years, when he contracted acute poliomyelitis which affected his arms and spinal muscles. He was treated in hospital for 9 months, during which time his right arm recovered some function, but his left arm remained useless. At the time of his discharge there was a mild scoliosis, and this later progressed until at the age of 13 years he was given a Milwaukee support. At this time he did not complain of dyspnoea, but his vital capacity was only $1100 \mathrm{ml}$.

He wore the spinal support for 5 years, but only intermittently in the latter part of this period and soon after discarding it completely he noticed increasingly severe dyspnoea. He was admitted to hospital for investigation.

On examination at the age of 18 he was not cyanosed and there were no signs of heart failure. There was a severe scoliosis convex to the right, and the left side of his chest moved much more with respiration than the right. The muscles of the arms, chest, and back were wasted. Vital capacity was $600 \mathrm{ml}$.

36 hours after his admission the boy became acutely ill with a respiratory infection. Staph. aureus was recovered from the sputum and the appropriate antibiotic therapy was prescribed. The boy became cyanosed and was treated in an oxygen tent, and appeared to be improving when suddenly he collapsed and died.

At necropsy the left diaphragm was 'paper thin' and transparent, no muscle fibres being visible. The right diaphragm was muscular, this part being $5 \mathrm{~mm}$ thick. The heart was said to be normal but for 'slight hypertrophy of the right ventricle', but no measurements were recorded.

This boy is known to have developed his scoliosis after the age of 5 years, and the deformity gradually progressed. Though his vital capacity was reduced at the age of 13 years, the boy did not complain of dyspnoea until a few months before his death. He was not cyanosed until 2 days before his death, and at no time were there signs of heart failure.

\section{REFERENCES}

Areechon, W., and Reid, L. (1963). Hypolasia of lung with congenital diaphragmatic hernia. British Medical fournal, 1, 230.

Bergofsky, E. H., Turino, G. M., and Fishman, A. P. (1959). Cardiorespiratory failure in kyphoscoliosis. Medicine, 38, 263.

Bucher, U., and Reid, L. (1961). Development of the intrasegmental bronchial tree: the pattern of branching and development of cartilage at various stages of intra-uterine life. Thorax, 16, 207.

Chapman, E. M., Dill, D. B., and Graybiel, A. (1939). The decrease in functional capacity of the lungs and heart resulting from deformities of the chest: pulmonocardiac failure. Medicine, 18, 167.

Coombs, C. F. (1930). Fatal cardiac failure occurring in persons with angular deformity of the spine. British fournal of Surgery, $18,326$.

Davies, G., and Reid, L. (1970). The growth of the alveoli and pulmonary arteries in childhood. Thorax, 25, 669.

Dunnill, M. S. (1962). Postnatal growth of the lung. Thorax, $17,329$.

Elliott, F. M. (1964). The pulmonary artery system in normal and diseased lungs: structure in relation to pattern of branching. Ph.D. Thesis, University of London.

Fischer, J. W., and Dolehide, R. A. (1954). Fatal cardiac failure in persons with thoracic deformities. Archives of Internal Medicine, 83, 687.

Fulton, R. M., Hutchinson, E. C., and Jones, A. M. (1952). Ventricular weight in cardiac hypertrophy. British Heart fournal, 14, 413.

Hippocrates (1849). On the articulations. In The genuine works of Hippocrates, vol. 2, p. 553. Trans, by F. Adams. Sydenham Society, London.

Kitagawa, M., Hislop, A., Boyden, E. A., and Reid, L. (1971). Lung hypoplasia in congenital diaphragmatic hernia: a quantitative study of airway, artery, and alveolar development. British fournal of Surgery, 58, 342.

Latham, P. M. (1846). Lectures on subjects connected with clinical medicine, comprising diseases of the heart, vol. 2, Lecture 29, pp. 219 and 223. Longman, London.

Lewis, C. S., Daines, M. C., Samuels, A. J., and Hecht, H. H. (1952). Cor pulmonale (pulmono-cardiac syndrome): a case report. Diseases of the Chest, 22, 261.

Naeye, R. L. (1961). Kyphoscoliosis and cor pulmonale: a study of the pulmonary vascular bed. American fournal of Pathology, 38, 561

Reid, L. (1966). Autopsy studies of the lungs in kyphoscoliosis. In Proceedings of a Symposium on Scoliosis, p. 71. Ed. by P. A. Zorab. National Fund for Research into Poliomyelitis and Other Crippling Diseases, London.

Correspondence to Professor Lynne Reid, Institute of Diseases of the Chest, Brompton Hospital, London S.W.3. 